\title{
ANALYTICAL PREDICTION AND EXPERIMENTAL MEASUREMENT FOR MODE CONVERSION AND SCATTERING OF PLATE WAVES AT NON-SYMMETRIC CIRCULAR BLIND HOLES IN ISOTROPIC PLATES
}

\author{
F.B. Cegla , A. Rohde^ and M. Veidt»
}

- Mechanical Engineering Department, Imperial College London, SW7 2AZ, UK

*Division of Mechanical Engineering, University of Queensland, Brisbane, Qld 4072, Australia

\begin{abstract}
A model for guided wave scattering from non-symmetric blind holes in isotropic plates using Poisson and Mindlin plate wave theories for in-plane and flexural wave modes, respectively, is presented. It makes use of the wave function expansion technique and coupling conditions at the defect boundary in order to evaluate the scattered far fields of the three fundamental guided wave modes. The results were compared to other analytical models as well as experimental measurements for mode conversion from SO to AO. Measurements agreed well with predictions confirming the validity of the model, highlighting at the same time the strong frequency dependence of the scattering and mode conversion behaviour.
\end{abstract}

Keywords: Plate waves, Scattering, Mode conversion

PACS: 43.20.Gp, 43.20.Mv, 43.35.Cg 


\section{INTRODUCTION}

The use of guided waves for the inspection of large plate like structures and their structural health monitoring is one of the key methods investigated in the development of advanced quantitative nondestructive evaluation (QNDE) techniques. Current guided wave systems can detect and locate damage [1]. However, to date other local inspection techniques still have to be applied at the damage location for quantitative non destructive evaluation.

Since guided waves are sensitive to most types of defects there is considerable interest in further development of guided wave techniques in order also to quantitatively evaluate the structural health of large plate like structures by means of permanent in-situ or detachable sensors. One of the most promising techniques in structural wave ultrasonic QNDE to this end is plate wave (diffraction) tomography [2], [3].

The inversion of guided wave data in tomography or any other technique requires accurate knowledge of the interaction of the wave with defects. Several models and studies have been presented. Diligent and Lowe have presented finite element and experimental results for the scattering of $S 0$ waves from through holes [4] and flat bottomed holes [5]. McKeon and Hinders [6] have also presented a model for $S 0$ scattering based on Kane-Mindlin plate theory. Wang and Chang [7] presented scattering models based on a wave function expansion technique as well as the Born approximation for the $S O$ and $A O$ modes using approximate plate wave theories (Kane-Mindlin). Vemula and Norris [8] presented models and solutions for scattering of flexural $A O$ waves from cylindrical defects in plates. Fromme and Sayir [9] also presented the modeling of $A O$ scattering by a through hole using Kirchhoff and Mindlin theory and near field measurements. They experimentally validated the better performance of Mindlin theory. 
The above studies predominantly concentrated on the modeling of scattering of the same mode as the incident mode and compare results to other models. Experimental data of guided wave scattering and particularly mode conversion at non symmetric defects is scarce. Grahn [10] is, to the authors' knowledge, the only one who has extensively addressed non-symmetric circular defects as well as mode coupling.

It is believed that even though at first sight the addition of mode coupling unnecessarily complicates analysis and experiments, there are substantial advantages to the approach. First of all non-symmetric damage is the most likely damage to be found in real life. Therefore mode coupling will almost always occur to some extent. Secondly the occurrence of mode coupling may substantially improve experimental results. Imagine the use of a transducer polarized to selectively excite one mode and a receiver that is sensitive only to a mode different to the incident mode. The receiver will hence pick up the mode conversion caused by a defect very strongly and thus be very sensitive to the defect properties. It is also convenient that with this approach the system is insensitive to the incident field and allows direct measurements of scatter field data without a background subtraction of the incident field that is commonly used and introduces a range of undesirable errors as pointed out by Konstantinidis et al. [11]. Alternative methods of identifying single modes in multi-mode signals as presented in [12] might also be helpful.

The purpose of this paper is to present an extension to Grahn's [10] model of guided wave scattering at part thickness, circular holes in isotropic plates. Approximate plate wave theories are used in order to keep computational time and complexity low. Compared to Grahn [10] Kirchhoff plate theory for flexural waves is substituted by Mindlin plate theory, which is a commonly accepted better model of the AO mode. The presented model thus is still numerically easy and fast to solve while it also shows an increased range of validity in 
the frequency-thickness and wavenumber-damage radius product domains. Furthermore in this paper experimental results are presented that show far field scattering measurements and validate the model.

\section{WAVE PROPAGATION MODELS}

The scattered fields are calculated using the wave function expansion method. In order to apply this technique the displacement and stress fields of the incident transmitted and scattered waves are expressed in the form of potential functions. A Bessel/Hankel series expansion with arbitrary coefficients is used to represent the wave fields and an adequate number of boundary conditions are used to evaluate the coefficients for the scattered and transmitted fields, the incident field being known.

The solution of the exact 3D elasto-dynamic equations has been described by Grahn [10]. Since however this approach is computationally very demanding, the approximate Poisson and Mindlin plate theories are used here for the fundamental compressional, shear and flexural wave modes respectively. The use of the approximate wave theories has the advantage of allowing numerically efficient and fast solutions.

In [10] Grahn presented a solution that coupled Poisson and Kirchhoff theory. The same approach is followed here, however Mindlin theory is substituted for Kirchhoff theory. The main features of the Mindlin model are based on the work presented by Vemula and Norris [8] for scattering of flexural waves from circular defects. Mindlin theory is a more complex but better model for flexural wave propagation in plates, therefore extending the range of frequencies for which the model is valid and improving its accuracy.

\subsection{Poisson theory}

The Poisson theory for a plate in plane stress is a simple approximation of the lowest order compressional wave in an infinite plate. The discourse of Achenbach [13] is followed here. 
The analysis is two-dimensional assuming a plate system in plane stress. The $x$ and $y$ coordinates define the plane with respective displacements $u$ and $v$.

The equations of motion for the plane stress system can be derived:

$$
\begin{aligned}
& \frac{\partial^{2} u}{\partial x^{2}}+\frac{1-v}{2} \frac{\partial^{2} u}{\partial y^{2}}+\frac{1+v}{2} \frac{\partial^{2} v}{\partial x \partial y}=\frac{\left(1-v^{2}\right) \rho}{E} \frac{\partial^{2} u}{\partial^{2} t} \\
& \frac{\partial^{2} v}{\partial y^{2}}+\frac{1-v}{2} \frac{\partial^{2} v}{\partial x^{2}}+\frac{1+v}{2} \frac{\partial^{2} u}{\partial x \partial y}=\frac{\left(1-v^{2}\right) \rho}{E} \frac{\partial^{2} v}{\partial t^{2}}
\end{aligned}
$$

where $E$ is Young's modulus of the material, $v$ is Poisson's ratio, $\rho$ is the density and $t$ is the time variable. Considering a uniform extensional displacement mode shape through the thickness in time harmonic form, $u=A e^{i(k x-\omega t)}, v=0$ it can be shown that the phase velocity of a compressional wave is $c_{p}=\frac{\omega}{k_{p}}=\sqrt{\frac{E}{\rho\left(1-v^{2}\right)}}$ with $\omega$ the circular frequency and $k_{p}$ the wavenumber of the wave. This shows that for Poisson theory the phase velocity is non-dispersive, i.e. constant over all frequencies.

When the displacement polarisation of the wave is turned by 90 degrees the analysis can be used to deduce the velocity of the fundamental shear horizontal plate mode. The time harmonic displacements now read: $u=0, v=A e^{i(k x-\omega t)}$. When used in equations (1) and (2) this yields a phase velocity of $c_{s}=\frac{\omega}{k_{s}}=\sqrt{\frac{E}{2 \rho(1+v)}}$ for the fundamental shear horizontal mode.

In the general case when both fundamental modes are present the displacement field can be expressed in the form of a scalar potential $\phi$ and a vector potential $\psi$. A cylindrical coordinate system is chosen here because of the geometry of the scatterer. The displacement field then takes the form of [13] 
$u_{r}=\frac{\partial \phi}{\partial r}+\frac{1}{r} \frac{\partial \psi}{\partial \theta}$ and $u_{\theta}=\frac{1}{r} \frac{\partial \phi}{\partial \theta}-\frac{\partial \psi}{\partial r}$.

Using the constitutive equations for linear elastic, isotropic materials [13] the stress fields can be evaluated.

$$
\begin{aligned}
& \sigma_{r r}=\frac{E}{1-v^{2}}\left[\frac{\partial^{2} \phi}{\partial r^{2}}+v\left(\frac{1}{r} \frac{\partial \phi}{\partial r}+\frac{1}{r^{2}} \frac{\partial^{2} \phi}{\partial \theta^{2}}\right)+\frac{E}{1+v}\left[\frac{1}{r} \frac{\partial^{2} \psi}{\partial r \partial \theta}-\frac{1}{r^{2}} \frac{\partial \psi}{\partial \theta}\right]\right] \text { and } \\
& \sigma_{r \theta}=\frac{E}{1+v}\left[\left(\frac{1}{r} \frac{\partial^{2} \phi}{\partial r \partial \theta}-\frac{1}{r^{2}} \frac{\partial \phi}{\partial \theta}\right)+\frac{E}{2(1+v)}\left[-\frac{\partial^{2} \psi}{\partial r^{2}}+\left(\frac{1}{r} \frac{\partial \psi}{\partial r}+\frac{1}{r^{2}} \frac{\partial^{2} \psi}{\partial \theta^{2}}\right)\right]\right]
\end{aligned}
$$

To determine the overall forces that act in the plane of the plate it is necessary to integrate the plate stresses over the thickness $2 h$ of the plate, $z \in[-h, h]$ as shown in Figure 1.

$$
\left(N_{r r}, N_{r \theta}\right)=\int_{-h}^{h}\left(\sigma_{r r}, \sigma_{r \theta}\right) d z
$$

In Poisson theory the fundamental extensional and shear modes do not create any out-ofplane displacements and moments in the plate. 
a)

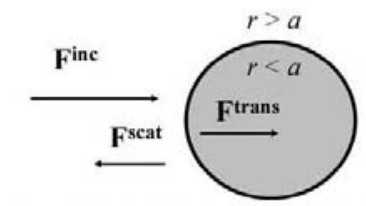

I

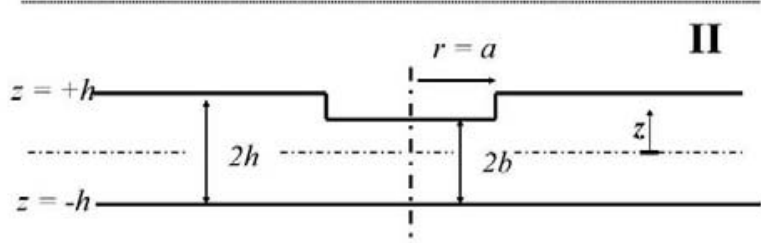

b)

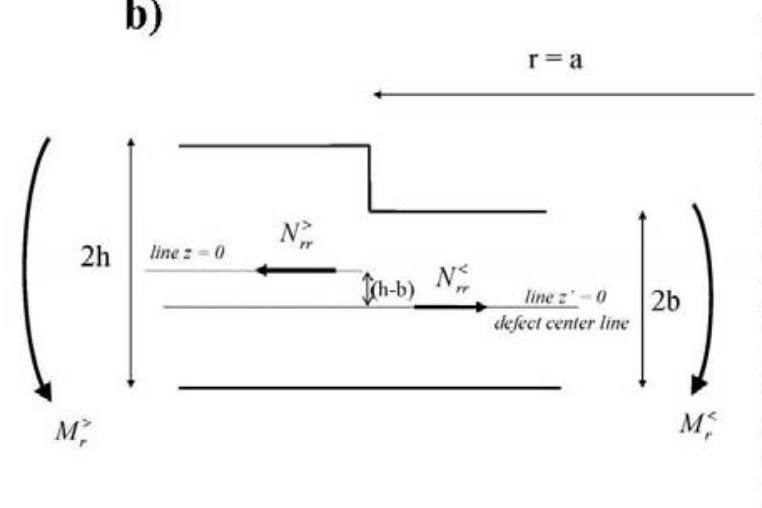

c)

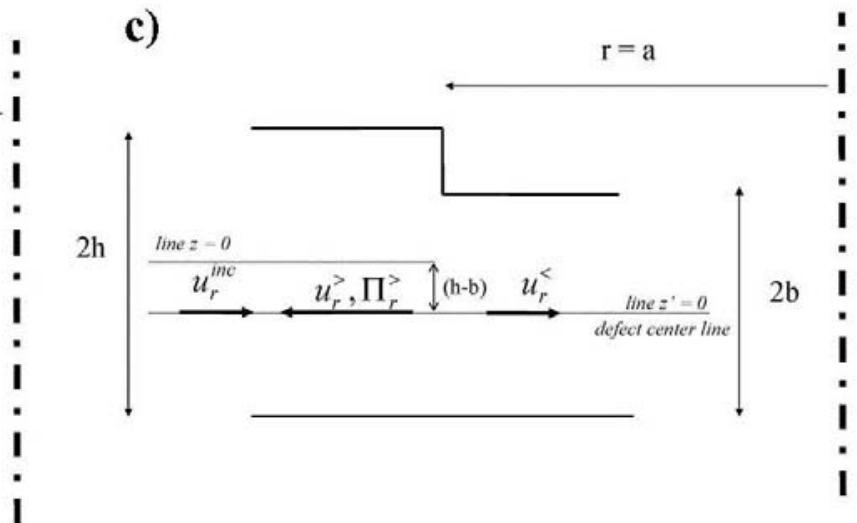

Figure 1 a) Schematic of the defect region: I plane view II cross-sectional view.

b) Schematic of the moments acting at the defect boundary.

c) Schematic of the radial displacements acting at the defect boundary.

\subsection{Mindlin theory}

While Poisson theory models the fundamental compressional and shear horizontal wave modes in a plate, Mindlin theory is a model of the fundamental flexural wave mode. Mindlin theory is discussed in detail by Graff [14] and Vemula and Norris [8], whose work is tightly followed here. In Mindlin theory the displacement components are expressed as follows:

$u=-z \Pi_{x}(x, y, t), v=-z \Pi_{y}(x, y, t)$ and $w=w(x, y, t)$.

where the plane of the plate is defined by $x$ and $y, u$ and $v$ are the in-plane displacements caused by the rotations $\Pi_{x}$ and $\Pi_{y}, z$ is the coordinate defining points across the thickness 
of the plate ( $z=0$ is the neutral plane) and $w$ is the out-of-plane displacement of the wave.

Three equations of motion can be obtained [8].

$$
\begin{aligned}
& \frac{D}{2}\left\{(1-v) \nabla^{2} \Pi_{x}+(1+v)\left(\frac{\partial^{2} \Pi_{x}}{\partial x^{2}}+\frac{\partial^{2} \Pi_{y}}{\partial x \partial y}\right)\right\}-\kappa^{2} \mu h\left(\Pi_{x}+\frac{\partial w}{\partial x}\right)-\frac{\rho h^{3}}{12} \frac{\partial^{2} \Pi_{x}}{\partial t^{2}}=0 \\
& \frac{D}{2}\left\{(1-v) \nabla^{2} \Pi_{y}+(1+v)\left(\frac{\partial^{2} \Pi_{y}}{\partial y^{2}}+\frac{\partial^{2} \Pi_{x}}{\partial x \partial y}\right)\right\}-\kappa^{2} \mu h\left(\Pi_{y}+\frac{\partial w}{\partial y}\right)-\frac{\rho h^{3}}{12} \frac{\partial^{2} \Pi_{y}}{\partial t^{2}}=0 \\
& \kappa^{2} \mu h\left(\nabla^{2} w+\frac{\partial \Pi_{x}}{\partial x}+\frac{\partial \Pi_{y}}{\partial y}\right)-\rho h\left(\frac{\partial^{2} w}{\partial t^{2}}\right)=0
\end{aligned}
$$

With the flexural rigidity $D=\frac{8 E h^{3}}{12\left(1-v^{2}\right)}, \mu$ is the shear modulus of the material and $\kappa$ a shear correction factor which is $\kappa^{2}=\frac{\pi^{2}}{12}$ for accurate representation of the low frequency behaviour (see Graff [14]).

Vemula and Norris [8] give expressions for the wavenumbers of the three different waves that arise in Mindlin theory:

$$
k_{1,2}^{2}=\frac{1}{2}\left(k_{p}^{2}+k_{t}^{2}\right) \pm \sqrt{k_{f}^{4}+\frac{1}{4}\left(k_{p}^{2}-k_{t}^{2}\right)^{2}} \text { and } k_{3}^{2}=\kappa^{2} \frac{k_{1}^{2} k_{2}^{2}}{k_{p}^{2}} \text {, }
$$

where

$$
k_{t}=\frac{\omega}{c_{t}}, c_{t}=\left(\frac{\kappa^{2} \mu}{\rho}\right)^{1 / 2}, k_{p}=\frac{\omega}{c_{p}}, k_{f}=\left(\frac{2 \rho h \omega^{2}}{D}\right)^{1 / 4} .
$$

Below the cutoff frequency of the flexural mode $A 1 k_{2}$ and $k_{3}$ are imaginary and represent evanescent waves while $k_{1}$ is the only real wavenumber and represents the propagating $A 0$ mode. 
Further Vemula and Norris [8] show that in Mindlin theory the out-of-plane displacement $w$ and rotations $\Pi_{r}, \Pi_{\theta}$ of a flexural wave can be expressed as a function of three independent potential functions $w_{1}, w_{2}$ and $V$. Again cylindrical coordinates are used here because of the cylindrical shape of the scatterer that is modelled.

These independent variables can be used to describe the out-of-plane displacement and the two rotations in the plate

$w(r, \theta)=w_{1}(r, \theta)+w_{2}(r, \theta)$

$\Pi_{r}=A_{1} \frac{\partial w_{1}}{\partial r}+A_{2} \frac{\partial w_{2}}{\partial r}+\frac{1}{r} \frac{\partial V}{\partial \theta}$ and $\Pi_{\theta}=A_{1} \frac{1}{r} \frac{\partial w_{1}}{\partial \theta}+A_{2} \frac{1}{r} \frac{\partial w_{2}}{\partial \theta}+\frac{\partial V}{\partial r}$

with $A_{j}=-1+\frac{k_{s}^{2}}{k_{j}^{2}}$. The potential functions fulfil the Helmholtz equations,

$\nabla^{2} w_{1}+k_{1}^{2} w_{1}=0, \nabla^{2} w_{2}+k_{2}^{2} w_{2}=0$ and $\nabla^{2} V+k_{3}^{2} V=0$.

(14a,b,c)

These quantities are similar to the potentials $\phi$ and $\psi$ in Poisson theory and the out-of-plane displacements $w_{1}$ and $w_{2}$ in Kirchhoff theory. In contrast to the simpler Kirchhoff theory however there are three independent potentials rather than only two.

Using the material constitutive equations, expressions for the moments and shear force can be derived, [8]

$$
\begin{aligned}
& M_{r}=D\left[\frac{\partial \Pi_{r}}{\partial r}+\frac{v}{r}\left(\frac{\partial \Pi_{\theta}}{\partial \theta}+\Pi_{r}\right)\right], M_{r \theta}=\frac{D}{2}(1-v)\left[\frac{\partial \Pi_{\theta}}{\partial r}+\frac{1}{r} \frac{\partial \Pi_{r}}{\partial \theta}-\frac{\Pi_{\theta}}{r}\right] \text { and } \\
& Q_{r}=\kappa^{2} h \mu\left(\frac{\partial w}{\partial r}+\Pi_{r}\right) .
\end{aligned}
$$




\section{SCATTERING MODEL}

\subsection{The defect region and wave fields}

The scattering of guided waves is considered here, therefore the background medium that wave propagation takes place in is assumed to be an infinite isotropic plate of uniform thickness $2 h$. Circular non-symmetric blind holes of radius $r=a$ and depth $2(h-b)$ are the defects that are modelled. A cylindrical coordinate system is defined with the origin at the centre of the defect. The region $r<a$ lies inside the circle and describes the interior of the defect while the area $r>a$ defines the infinite plate surrounding the defect. The material properties within both regions are assumed to be uniform. Figure 1 shows the defect geometry.

By means of arrows Figure 1a also indicates the wave fields that are to be expected in the plate. At the discontinuity (scatterer) the incoming wave field will be partly transmitted into the defect and also partly scattered back into the surrounding plate. Mode conversion can occur so that not only the incoming mode but also other modes can be part of the scattered and transmitted wave fields. From Figure 1a it is obvious that at the boundary between the two regions, i.e. $r=a$, for any field quantity $F$ the sum of the incoming and scattered fields equals the transmitted field.

$F^{\text {inc }}+F^{\text {scat }}=F^{\text {trans }}$ at $r=a$.

Since approximate theories are used for the guided wave modes it has to be stressed here that a particular meaning is attached to the term 'field quantity'. For example the $S 0$ and $S H$ modes have direct contributions to the in-plane displacements while the $A 0$ mode contributes to in-plane displacements via its rotations.

$F^{i n c}$ is entirely known and assumed to be a plane $S 0$ wave. In order to set up equations to solve for the scattered and transmitted parts it is convenient to make use of Bessel or Hankel series expansions of the potentials of the wave fields. Only monochromatic waves 
are considered in the scattering model, so that the time factor $e^{i \omega t}$ can be omitted in the analysis.

The Bessel/Hankel series expansions can describe any type of wave field in cylindrical coordinates. While the series coefficients at each circumferential order are known for the incoming field they will have to be evaluated for the scattered and transmitted fields. For the evaluation of these coefficients the boundary and continuity conditions are needed. The expansions for the potentials of Poisson theory can be expressed as [10]:

$\phi^{>}(r, \theta)=h \sum_{m=-\infty}^{m=\infty} a_{m} H_{m}\left(k_{p}^{h} r\right) e^{i m \theta}, \quad \phi^{<}(r, \theta)=h \sum_{m=-\infty}^{m=\infty} f_{m} J_{m}\left(k_{p}^{b} r\right) e^{i m \theta}$

and

$\psi^{>}(r, \theta)=h \sum_{m=-\infty}^{m=\infty} b_{m} H_{m}\left(k_{s}^{h} r\right) e^{i m \theta}, \psi^{<}(r, \theta)=h \sum_{m=-\infty}^{m=\infty} g_{m} J_{m}\left(k_{s}^{b} r\right) e^{i m \theta}$

where the signs $>$, $<$ stand for the outwardly $(r>a)$ and inwardly $(r<a)$ radiating potentials respectively and the superscripts $h$ and $b$ indicate whether the wavenumber is evaluated for the thickness of the plate or the defect. $a_{m}, b_{m}, f_{m}$ and $g_{m}$ are the unkown coefficients of the expansion. The symbols $J_{m}$ and $H_{m}$ represent the Bessel function of order m and Hankel function of the first kind of order $m$ respectively. Only monochromatic waves are considered so that the time factor $e^{i \omega t}$ can be omitted. A Bessel series expansion is used to model the field within the defect region while a Hankel series expansion is used for the scattered field. The different functions are used for a correct representation of the field and in order to avoid singularities.

Similarly, the potentials for Mindlin theory are:

$$
w_{1}^{>}(r, \theta)=\sum_{m=-\infty}^{m=\infty} c_{m} H_{m}\left(k_{1}^{h} r\right) e^{i m \theta}, w_{1}^{<}(r, \theta)=\sum_{m=-\infty}^{m=\infty} h_{m} J_{m}\left(k_{1}^{b} r\right) e^{i m \theta}
$$




$$
\begin{aligned}
& w_{2}^{>}(r, \theta)=\sum_{m=-\infty}^{m=\infty} d_{m} H_{m}\left(k_{2}^{h} r\right) e^{i m \theta}, w_{2}^{<}(r, \theta)=\sum_{m=-\infty}^{m=\infty} g_{m} J_{m}\left(k_{2}^{b} r\right) e^{i m \theta} \\
& V^{>}(r, \theta)=\sum_{m=-\infty}^{m=\infty} e_{m} H_{m}\left(k_{3}^{h} r\right) e^{i m \theta}, V^{<}(r, \theta)=\sum_{m=-\infty}^{m=\infty} i_{m} J_{m}\left(k_{3}^{b} r\right) e^{i m \theta}
\end{aligned}
$$

where the signs $>$, < again indicate outwardly or inwardly radiating potentials, $c_{m}, d_{m}, e_{m}$, $h_{m}, g_{m}$ and $i_{m}$ are the unkown coefficients of the expansion and superscripts $h$ and $b$ indicate the thickness of the plate at the local position.

Equations (18)-(22) show that there are 10 unknown coefficients at each circumferential order $m$ of the problem, 5 unknown coefficients (2 Poisson theory, 3 Mindlin theory) for the scattered field $(>)$ and 5 unknown coefficients for the transmitted field $(<)$. Due to the orthogonality of circumferential orders equations can be set up at each integer circumferential order. In total there are 10 equations and 10 unknowns to be determined at each circumferential order.

For each unknown a boundary condition must be defined so that a set of simultaneous equations can be set up and solved. While the exact solution would consist of the summation of an infinite number of circumferential orders $\mathrm{m}$, in practice it is found that the solution quickly converges and only the lowest circumferential orders are significant, the number of terms being dependent on the size of the hole relative to the wavelength. All the solutions presented in this paper converged after evaluations of the lowest 10 circumferential orders.

The incoming wave field is described using a Bessel series expansion. In case of an incident plane wave $S 0$ mode the incoming field can be deduced from the potentials [10]

$$
\phi(r, \theta)=h \sum_{m=-\infty}^{m=\infty} i^{m} J_{m}\left(k_{p} r\right) e^{i m \theta} \text { and } \psi(r, \theta)=0
$$

The displacement and stress fields of the incident wave follow from these expressions together with equations (3a,b)-(5). 


\subsection{Boundary conditions}

Continuity of the wave fields across the defect boundary is used to formulate the boundary conditions. However before moving on to the actual definition of the boundary conditions it is important to note at this point that the definition of the boundary conditions will also directly describe the mode coupling between the two theories. The neutral axis of a nonsymmetric defect region is offset from the neutral axis of the plate material surrounding it. In a static approximation it can be seen that this creates a moment due to the offset of the in-plane forces acting in the plate. Since the incident $S 0$ wave does not contain any moments, a flexural wave is created when the wave interacts with the discontinuity. An illustration of the balance of moments is shown in Figure 1b. Figure 1c further shows a balance of the displacements at the defect boundary.

Using continuity conditions for all displacements, forces and moments as well as the coupling conditions illustrated in Figure $1 \mathrm{~b}$ and Figure 1c the following equations are derived:

Radial and angular in-plane displacements

$u_{r}^{>}-u_{r}^{<}+(h-b) \Pi_{r}^{>}=-u_{r}^{i n c} \quad$ and $\quad u_{\theta}^{>}-u_{\theta}^{<}+(h-b) \Pi_{\theta}^{>}=-u_{\theta}^{i n c}$

Out-of-plane displacements

$w^{>}-w^{<}=0$

Radial and angular rotation

$$
\Pi_{r}^{>}-\Pi_{r}^{<}=0 \quad \text { and } \quad \Pi_{\theta}^{>}-\Pi_{\theta}^{<}=0
$$

Radial and angular in-plane forces

$$
N_{r r}^{>}-N_{r r}^{<}=-N_{r r}^{i n c} \quad \text { and } \quad N_{r \theta}^{>}-N_{r \theta}^{<}=-N_{r \theta}^{i n c}
$$

Radial and angular moments 
$M_{r}^{>}-M_{r}^{<}+(h-b) N_{r r}^{<}=0 \quad$ and $\quad M_{\theta}^{>}-M_{\theta}^{<}+(h-b) N_{r \theta}^{<}=0$

(28a,b)

Radial shear forces

$Q_{r}^{>}-Q_{r}^{<}=0$

\subsection{Matrix Equation}

By substitution of the Hankel and Bessel series expansions into the boundary condition equations (24a,b)-(29) a set of 10 simultaneous equations is obtained. Due to the orthogonality of the expansions the equations can be formulated at each circumferential order $m$ according to the following matrix equation:

$\left[\begin{array}{cccccccccc}\alpha_{1,1} & \alpha_{1,2} & \alpha_{1,3} & \alpha_{1,4} & \alpha_{1,5} & \alpha_{1,6} & \alpha_{1,7} & 0 & 0 & 0 \\ \alpha_{2,1} & \alpha_{2,2} & \alpha_{2,3} & \alpha_{2,4} & \alpha_{2,5} & \alpha_{2,6} & \alpha_{2,7} & 0 & 0 & 0 \\ 0 & 0 & \alpha_{3,3} & \alpha_{3,4} & 0 & 0 & 0 & \alpha_{3,8} & \alpha_{3,9} & 0 \\ 0 & 0 & \alpha_{4,3} & \alpha_{4,4} & \alpha_{4,5} & 0 & 0 & \alpha_{4,8} & \alpha_{4,9} & \alpha_{4,10} \\ 0 & 0 & \alpha_{5,3} & \alpha_{5,4} & \alpha_{5,5} & 0 & 0 & \alpha_{5,8} & \alpha_{5,9} & \alpha_{5,10} \\ \alpha_{6,1} & \alpha_{6,2} & 0 & 0 & 0 & \alpha_{6,6} & \alpha_{6,7} & 0 & 0 & 0 \\ \alpha_{7,1} & \alpha_{7,2} & 0 & 0 & 0 & \alpha_{7,6} & \alpha_{7,7} & 0 & 0 & 0 \\ 0 & 0 & \alpha_{8,3} & \alpha_{8,4} & \alpha_{8,5} & \alpha_{8,6} & \alpha_{8,7} & \alpha_{8,8} & \alpha_{8,9} & \alpha_{8,10} \\ 0 & 0 & \alpha_{9,3} & \alpha_{9,4} & \alpha_{9,5} & \alpha_{9,6} & \alpha_{9,7} & \alpha_{9,8} & \alpha_{9,9} & \alpha_{9,10} \\ 0 & 0 & \alpha_{10,3} & \alpha_{10,4} & \alpha_{10,5} & 0 & 0 & \alpha_{10,8} & \alpha_{10,9} & \alpha_{10,10}\end{array}\right]\left[\begin{array}{c}a_{m} \\ b_{m} \\ c_{m} \\ d_{m} \\ e_{m} \\ f_{m} \\ g_{m} \\ h_{m} \\ i_{m} \\ j_{m}\end{array}\right]=\left[\begin{array}{c}\beta_{1} \\ \beta_{2} \\ 0 \\ 0 \\ 0 \\ \beta_{6} \\ \beta_{7} \\ 0 \\ 0 \\ 0\end{array}\right]$

In equation (30) the matrix contains the known information about the scattered and transmitted wave fields, the first column vector contains the unknown coefficients of the potential functions and the second column vector contains information about the incident field. The individual terms of $\alpha_{i, j}$ and $\beta_{i}$ are given in the appendix.

The matrix equation (30) can easily be solved numerically by Gaussian elimination or similar algorithms.

\subsection{Scattered field solutions}


Due to mode conversion the pure incident mode will be scattered into all three fundamental guided wave modes. By solving for the unknowns the amplitudes of all three modes can be evaluated in the far field. The amplitudes of the dominant displacement component for each mode ( $U_{r}$ for $S 0, U_{\theta}$ for $S H, U_{z}$ for $A 0$ ) are computed on a radius $r$ around the scatterer using equations (3a,b) and (12). In order to compare the relative amplitudes of these displacements they are normalised to the maximum of the incident displacement field as follows.

$$
U_{r, \theta, z}^{\infty}(\theta)=\frac{\left|u_{r, \theta, z}(r, \theta)\right|}{\max \left(\left|u_{r}^{\text {inc }}(a, \theta)\right|\right)} \sqrt{\frac{r}{h}} \text { as } r \rightarrow \infty .
$$

Note here that the $\sqrt{ }(r / h)$ term enters the expression to correct for geometric decay of the wave field with increased distance from the scatterer. The expression in equation (31) is convenient since it enables direct comparison with Grahn's solutions in [10].

The results computed in equations (31) give a good estimate of the far field directivity of the scattered modes with scaled amplitudes. Since all displacement amplitudes are scaled with respect to the incoming $S O$ wave and the in-plane and flexural stiffnesses of a plate normally differ by two orders of magnitude this can cause values greater than 1 for the scattered amplitudes. It has to be noted that this is a result of scaling rather than a violation of energy conservation. Another comparison would be the evaluation of the scattered fields on the rim of the defect $(r=a)$ normalised to the incident field. However these fields cannot be measured in practice, since they are superposed onto evanescent mode fields that cause local perturbations. Evanescent wave fields are not correctly accounted for in the approximate theories.

The Kirchhoff theory predicts the presence of one evanescent mode ( $k_{2}$ is imaginary) and Mindlin theory 2 evanescent modes ( $k_{2}, k_{3}$ are imaginary) however whether these are the dominant non-propagating modes involved in the scattering process is unknown. Many 
evanescent modes are involved in the scattering process (see Diligent [15]) some of which are dominant depending on the geometry of the problem. Since the approximate theories may result in a poor representation of the evanescent fields the normalised far field representation according to equation (31) is used in the comparisons.

\section{COMPARISON TO OTHER MODELS}

The modelling approach outlined in this paper has closely followed that of Grahn [10], therefore an initial comparison of the present model results to his 3D-equation model and the approximate Poisson/Kirchhoff model was used as a first validation step. As an illustration, model results for different dimensionless frequencies $\Omega=\omega h / c_{T}$ and damage depths were obtained for the Mindlin model that was presented here and were compared to Grahn's published results for the Kirchhoff and 3D exact equation models.

The comparisons are shown in Figure 2-Figure 4. It can be seen that there is good agreement between all three models at low frequencies $(\Omega=0.06$, Figure 2$)$. When the frequency is increased ( $\Omega=0.4$, Figure 3) both the Mindlin and the Kirchhoff model start to deviate from the 3D exact solution, however the Mindlin model outperforms the Kirchhoff model. Notable deviations only occur in the $A 0$ scattered field. The scatter pattern of the Mindlin solution still represents the 3D exact solution relatively well (within 10-15\%) while the Kirchhoff $A 0$ scatter pattern differs considerably from both of the other solutions (differences of up to 50\%).

At even higher frequencies ( $\Omega=1.2$, Figure 4 ) the scattered field of all three modes are wrongly predicted by the Kirchhoff model. There are also larger deviations in amplitude between the Mindlin model and the 3D exact model (differences up to 25\%), however the shape of the scatter pattern (number of lobes and directivity) of both models still agrees, despite deviations in amplitude. 


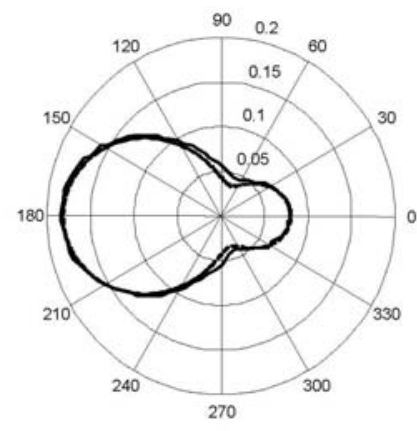

$\mathrm{U}_{\mathrm{r}}$

a) Scattered S0 wave

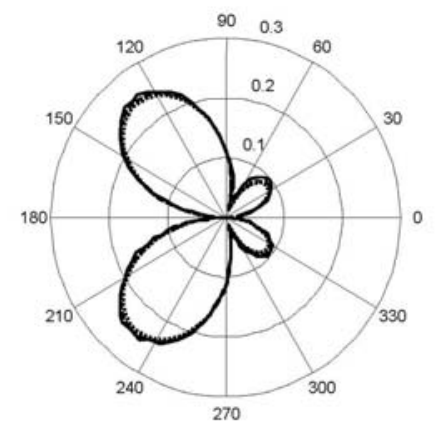

$\mathrm{U}_{\theta}$

b) Scattered SH wave

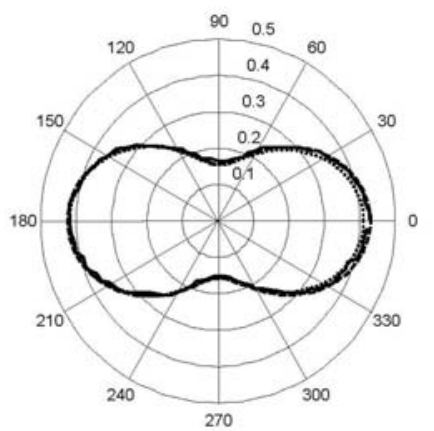

$\mathrm{U}_{\mathrm{z}}$

c) Scattered A0 wave

Figure 2 Grahn's 3D exact model results (-), Poisson/Kirchhoff model results ( $\cdots$ ) and Poisson/Mindlin model results (---) for a hole of radius $a / h=4$ and depth $b / h=0.2$ in a steel plate at dimensionless frequency $\Omega=\omega h / c_{T}=0.06$.

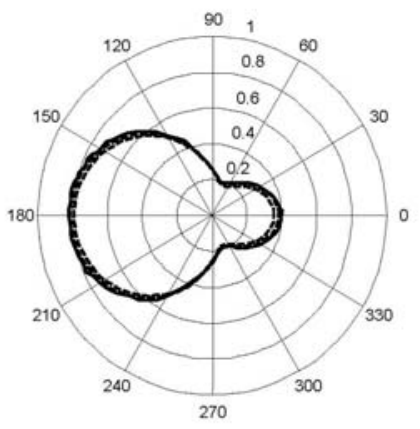

$\mathrm{U}_{\mathrm{r}}$

a) Scattered S0 wave

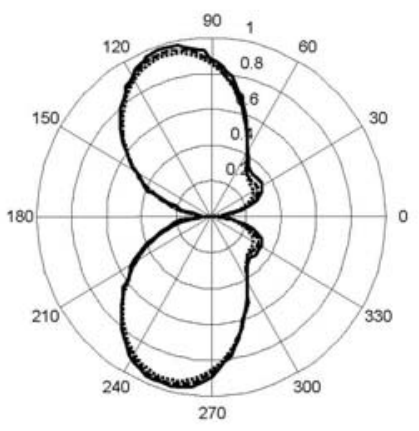

$\mathrm{U}_{\theta}$

b) Scattered SH wave

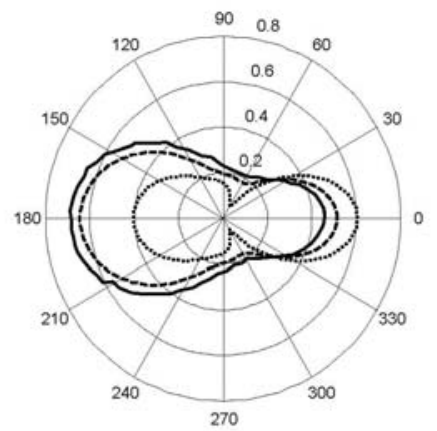

$\mathrm{U}_{\mathrm{z}}$

c) Scattered A0 wave

Figure 3 Grahn's 3D exact model results (-), Poisson/Kirchhoff model results (..$)$ and Poisson/Mindlin model results (---) for a hole of radius $a / h=4$ and depth $b / h=0.5$ in a steel plate at dimensionless frequency $\Omega=\omega h / c_{T}=0.4$.

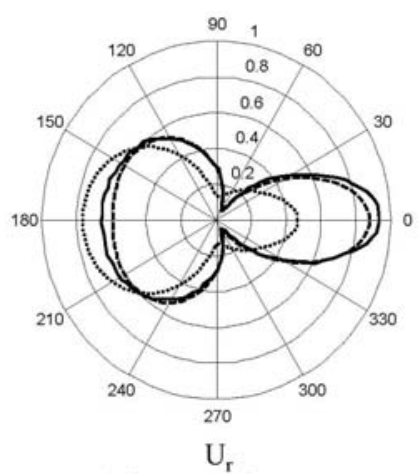

a) Scattered S0 wave

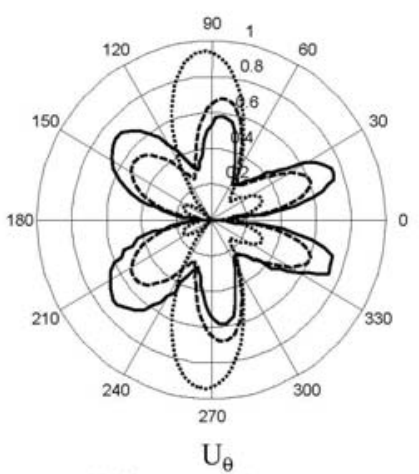

b) Scattered $\mathrm{SH}$ wave

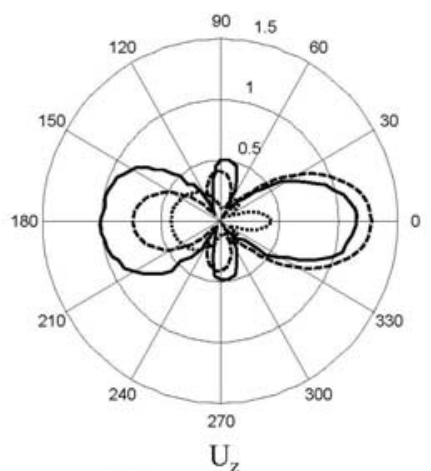

c) Scattered A0 wave

Figure $4 \quad$ Grahn's 3D exact model results (-), Poisson/Kirchhoff model results ( $\cdots$ ) and Poisson/Mindlin model results (---) for a hole of radius $a / h=4$ and depth $b / h=0.5$ in a steel plate at dimensionless frequency $\Omega=\omega h / c_{T}=1.2$.

\section{EXPERIMENTAL VALIDATION}

\subsection{Setup}


The equipment used to test the model consisted of a $1.2 \mathrm{~mm}$ thick aluminium plate (length $1.2 \mathrm{~m}$, width $1 \mathrm{~m}, E=70.7 \mathrm{GPa}, \rho=2700 \mathrm{~kg} / \mathrm{m}^{3}, v=0.33$, experimentally verified). A piezo electric transducer (length $12 \mathrm{~mm}$, width $3 \mathrm{~mm}$, thickness $2 \mathrm{~mm}$, PZT27 Ferroperm) was bonded to the cross section of the plate with superglue in order to excite the fundamental SO plate mode.

The signal was generated by a computer controlled arbitrary waveform generator (Stanford instruments Inc.) which outputs a $10 \mathrm{~V}$ peak to peak, 5 cycle Hanning windowed toneburst at the desired center frequency. Amplification of the excitation signal by a factor of 10-50 was then achieved by use of a power amplifier (Krohn Hite model 7500). The output of the amplifier was connected across the electrodes of the piezo crystal.

Flat bottomed blind hole defects of radius $6,7.5$ and $10 \mathrm{~mm}$ and $0.6 \mathrm{~mm}$ depths were introduced in the plate at about 700mm from the transducer location.

A laser vibrometer (OFV 303/OFV 3001, Polytech GmbH) was used to measure the out-ofplane displacements at points on a radius of $50 \mathrm{~mm}$ around the centre of the defects. The laserhead was positioned by a computer controlled positioning system (Newport ESP 300) and scanned along the radius from $0^{\circ}$ to $180^{\circ}$ in $15^{\circ}$ steps. Since the field is expected to be symmetric, only one half was scanned.

The detected out-of-plane displacement time trace from the laser vibrometer was fed into a PC via a digital acquisition card (NI PCI 6251) and was stored. The setup is shown in Figure 5. Figure 6 shows a typical set of timetraces recorded by the system before and after a defect was introduced. The signals show that the incident $S 0$ wave, which is expected to arrive at approximately $0.2 \mathrm{~ms}$, is not visible for the laser vibrometer but a clear, directionally dependent SO-AO mode converted wave pulse is observed in the case of the 'damaged' plate. In both the 'damaged' and 'undamaged' plate cases also an $A 0$ signal arrival is detected. The $A O$ mode is excited by the transducer due to transverse 
electromechanical coupling in the transducer, misalignments in its attachment and the low flexural compared to the in-plane stiffness of the plate. However since it arrives later than the SO-AO mode converted signal it can be gated out and removed from the data without problems.

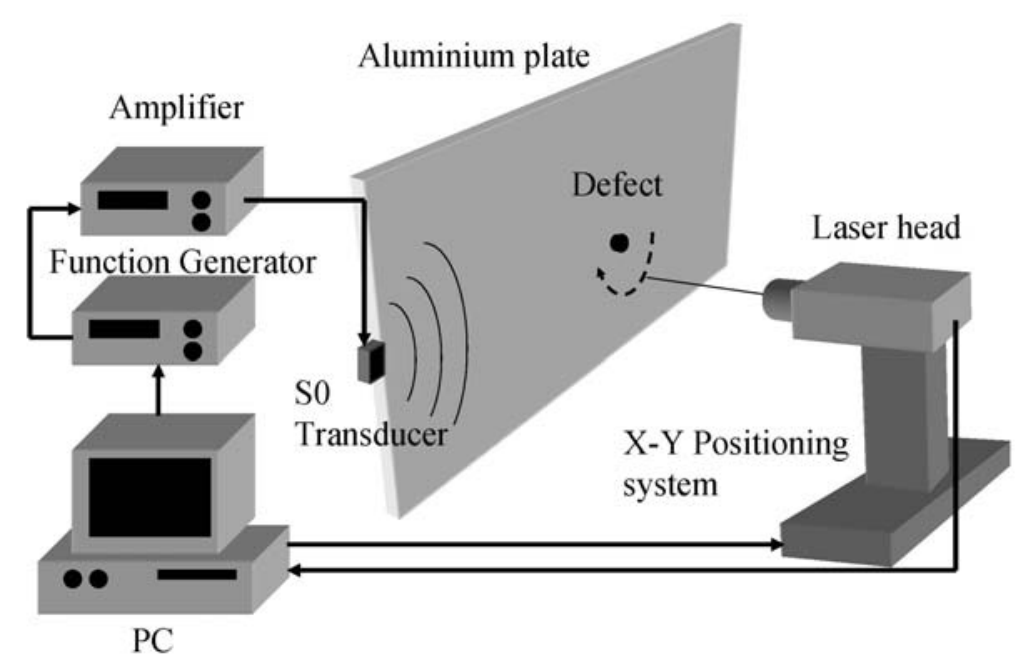

Figure 5 Schematic of the experimental setup.
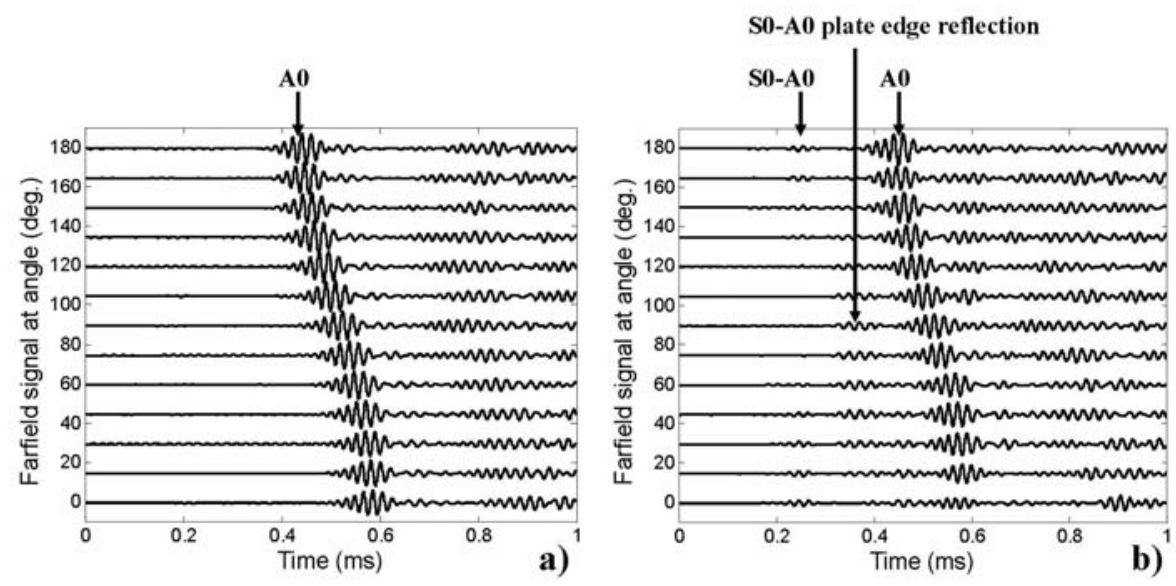

Figure 6 Example set of timetraces collected for a) a plate without defect and b) a plate with half thickness blind hole defect.

To obtain a polar directivity plot of the mode converted $A O$ wave field the $S 0$ - $A 0$ mode converted signal was gated. Then the maximum absolute amplitude was determined in the 
time domain. The maximum values within each gated timetrace were then plotted against the angular position to obtain the directivity plot that could be compared to the model results. Each plot was normalized to the maximum amplitude value within itself.

\subsection{Results and Discussion}

The measured far field scatter patterns for the three different blind holes are shown in Figure 7 - Figure 9. The measurements were taken at 3 different centre frequencies. Only the out-of-plane displacement component was measured and is shown. Model predictions from the Poisson/Mindlin (solid line) and Poisson/Kirchhoff (dashed line) models are also shown. The solid markers indicate measurements at a distance of $50 \mathrm{~mm}$ from the centre of the scatterer.

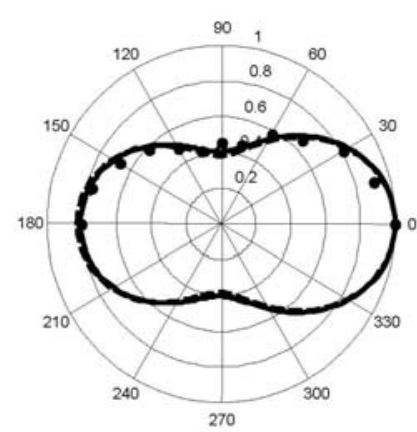

$\mathrm{U}_{\mathrm{z}}$

a) $\mathrm{A} 0$ scatter $30 \mathrm{kHz}$

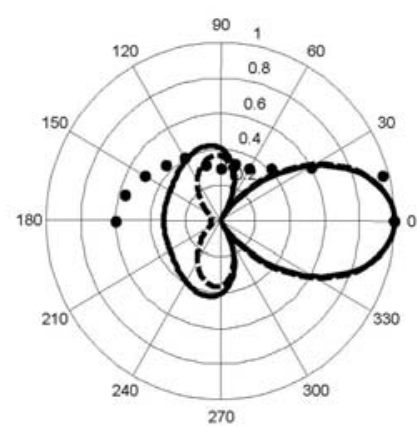

$\mathrm{U}_{\mathrm{Z}}$

b) A0 scatter $50 \mathrm{kHz}$

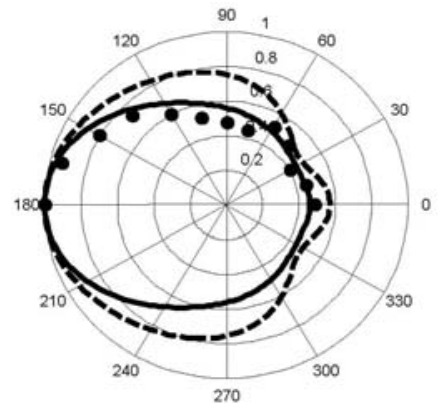

$\mathrm{U}_{\mathrm{z}}$

c) $\mathrm{A} 0$ scatter $70 \mathrm{kHz}$

Figure 7 Amplitude of the $A 0$ field scattered by a $\varnothing 12 \mathrm{~mm}(\mathrm{a} / \mathrm{h}=10)$ blind hole of $0.6 \mathrm{~mm}$ $(b / h=0.5)$ depth measured on a radius $(\bullet) R=50 \mathrm{~mm}$ from the centre of the defect compared to model predictions (--- Kirchhoff, - Mindlin) at different frequencies. 


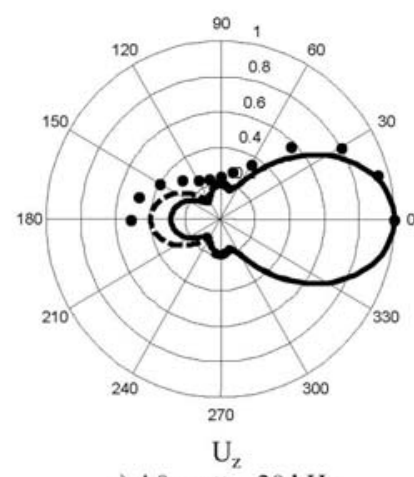

a) $\mathrm{A} 0$ scatter $30 \mathrm{kHz}$

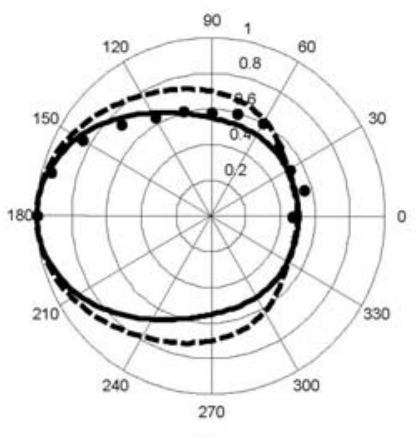

$\mathrm{U}_{\mathrm{z}}$

b) A0 scatter $50 \mathrm{kHz}$

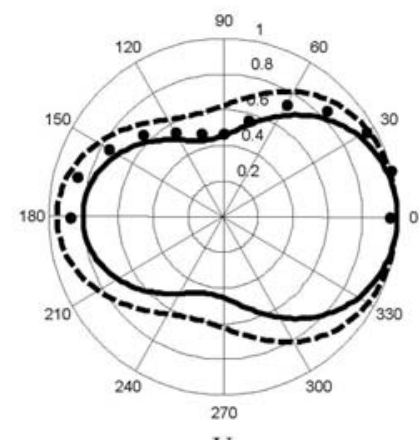

c) $\mathrm{A} 0$ scatter $70 \mathrm{kHz}$

Figure 8 Amplitude of the $A 0$ field scattered by a $\varnothing 15 \mathrm{~mm}(a / h=12.5)$ blind hole of $0.6 \mathrm{~mm}(b / h=0.5)$ depth measured on a radius $(\bullet) R=50 \mathrm{~mm}$ from the centre of the defect compared to model predictions (--- Kirchhoff, — Mindlin) at different frequencies.

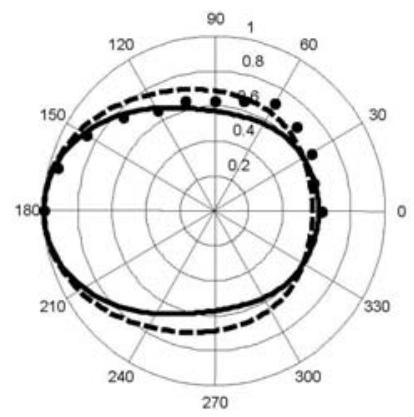

$\mathrm{U}_{2}$

a) A0 scatter $30 \mathrm{kHz}$

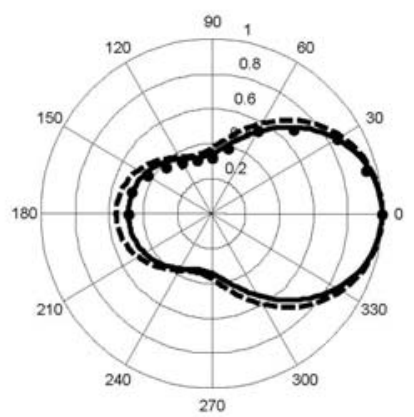

$\mathrm{U}_{\mathrm{z}}$

b) $\mathrm{A} 0$ scatter $50 \mathrm{kHz}$

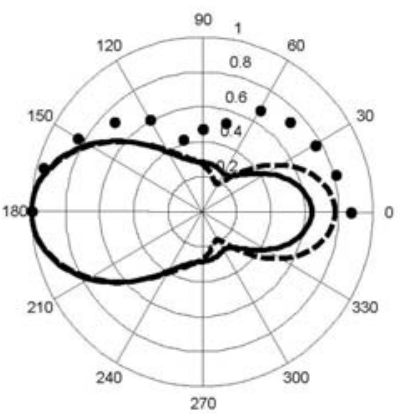

$\mathrm{U}_{\mathrm{z}}$

c) $\mathrm{A} 0$ scatter $70 \mathrm{kHz}$

Figure 9 Amplitude of the $A 0$ field scattered by a $\varnothing 20 \mathrm{~mm}(a / h=16.7)$ blind hole of $0.6 \mathrm{~mm}(b / h=0.5)$ depth measured on a radius $(\bullet) R=50 \mathrm{~mm}$ from the centre of the defect compared to model predictions (--- Kirchhoff, — Mindlin) at different frequencies.

Figure 6 shows that the mode conversion from $S 0$ to $A 0$ at the defect is a convenient way to measure the scattered $A 0$ field directly since the incident mode is barely received by the laser vibrometer while it is very sensitive to the scattered mode. The measurement results in Figure 7 - Figure 9 further show that the presented model correctly predicts the scattered AO field. While there are some discrepancies (e.g. Figure $7 \mathrm{~b}$ ) between the measured and predicted fields, the model correctly predicts overall changes in the directions of the scatter with changes in frequency and hole diameter. 
The discrepancies that can be noted in Figure 7b, Figure 8a and Figure 9c are mainly due to a reduced amplitude of the scattered signal and the accompanied higher noise levels in the measurements. For specific frequencies and hole diameters $(12 \mathrm{~mm}, 50 \mathrm{kHz} ; 15 \mathrm{~mm}, 30 \mathrm{kHz}$; $20 \mathrm{~mm}, 70 \mathrm{kHz}$ ) the amplitude of the predicted scattered field drops to a minimum as shown in Figure 10. It is believed that at angular positions where the scattered amplitude is very low the system encounters noise levels that are stronger than the scattered field and distortion of the measurements can occur.

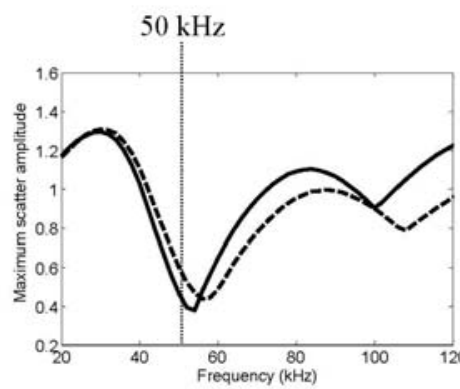

a) $\varnothing 12 \mathrm{~mm}$ Hole

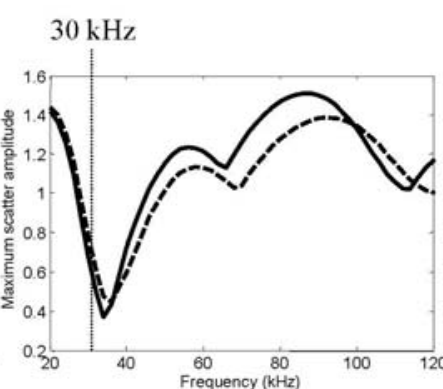

b) $\varnothing 15 \mathrm{~mm}$ Hole

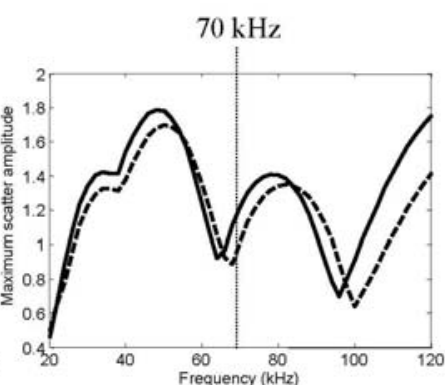

c) $\varnothing 20 \mathrm{~mm}$ Hole

Figure 10 Maximum predicted far field $A 0$ scatter amplitude around the defect as a function of frequency for a) a $\varnothing 12 \mathrm{~mm}$, b) a $\varnothing 15 \mathrm{~mm}$ and c) a $\varnothing 20 \mathrm{~mm}$ hole of depth $0.6 \mathrm{~mm}$ according to Mindlin (-) and Kirchhoff (---) theory.

The results also suggest that the measurements agree slightly better with the scatter patterns predicted by Mindlin theory rather than Kirchhoff theory. Both theories predict the same patterns at low frequencies and hole diameters, however for larger hole diameters and higher frequencies there are discrepancies. Ignoring the results where the scatter amplitude is weak (Figure 7b, Figure 8a, Figure 9c) it can be noted that as expected at higher frequencies and larger diameters (e.g. Figure 7c, Figure 8c, Figure 9b) the measurements appear to be closer to the patterns described by Mindlin theory.

Figure 11 shows the received timetraces for a $20 \mathrm{~mm}$ diameter hole. It is interesting to note in this figure that the signals that have been backscattered at 180 degrees contain more 
cycles than those that are forward scattered at 0 degrees. It is believed that this is due to interference of the scattered waves from the front and back ends of the defect. The scattered signals of both edges interfere constructively or destructively with each other and produce a signal with a different amount of cycles. This is a manifestation of reverberations of the wave within the defect. Since the modelling with the wave function expansion technique assumes steady state monochromatic waves, this phenomenon indicates that comparison of the time domain pulsed experimental data with the frequency domain model results could become inadequate when the wavelength becomes larger. Phase changes between the reflections of the front and back end of the defect become important and interference of the two signals can considerably distort the pulse shape. However the presented experimental results still matched the model reasonably well. Reverberation effects are likely to reduce for less severe damage than the half thickness blind holes used here.

An interesting ground for future work is the exploration of the discrepancies between experiment and model. This would most likely entail a detailed study of scattering of transient waves as compared to monochromatic waves as presented here. The conversion of the monochromatic frequency domain data into the time domain as well as comparison to time domain numerical simulations as such described by [16] would be of interest.

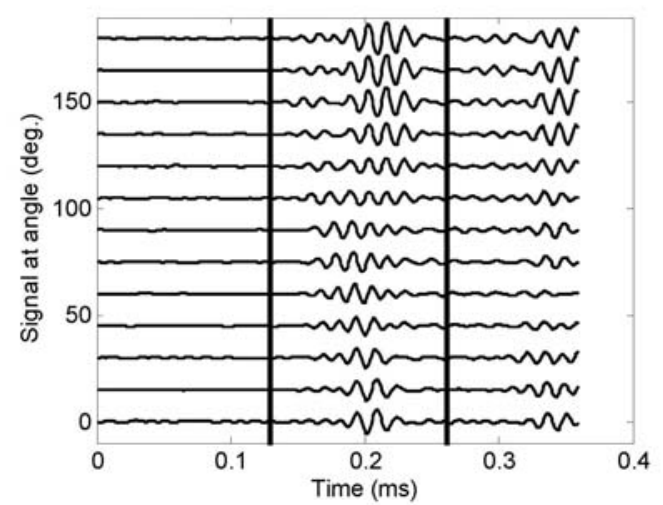

Figure $11 S 0$-A0 mode converted signals received from the $\varnothing 20 \mathrm{~mm}$ hole at $70 \mathrm{kHz}$ including the gate that was used for data processing. 


\section{CONCLUSION}

A model for predicting guided wave scattering from non-symmetric blind holes in plates using the approximate Poisson theory for compressional and shear waves and Mindlin theory to model flexural waves has been presented. The model uses the wave function expansion technique to evaluate the scattered fields. On comparison to an existing approximate model using Poisson/Kirchhoff theory and a model that uses the exact 3D equations it was found that the Poisson/Mindlin model performs better especially at higher frequencies than the Poisson/Kirchhoff model at the cost of a marginal increase of complexity. The computation of the exact 3D equations is more complicated and demanding on computational time making its use impractical.

Experimental results showed that $S O-A O$ mode converted data can easily be acquired and that the presented direct measurement of a mode converted scattered field is a substantial improvement compared to conventional methods that use background subtraction of the incident field to reveal the scattered field. Good measurement results showed that model predictions agreed with experimental measurements. Whereas predictions using Poisson/Mindlin theory seemed to have a slight edge over Poisson/Kirchhoff theory predictions, the difference between both model predictions was small for the hole diameters and frequencies that were investigated.

Careful examination of the collected time traces showed that for larger hole diameters and higher frequencies the number of cycles within the scattered signal was increased or decreased depending on the direction of scatter. It was believed that this is due to the interference of the signals scattered from the front and back of the defect rim.

Since the model assumes monochromatic waves and experimental results were collected using pulsed wave packets discrepancies between the two results can arise. Future work 
will address these issues and extend the model to include transient waves as well as analyse experimental data in the frequency domain.

\section{ACKNOWLEDGEMENTS}

The work was supported by the Australian Research Council under Discovery Project DP0344955. Portions were also supported by the Defence Science Technology Organisation under agreement B04/10. 


\section{APPENDIX - Mindlin matrix elements}

The elements of matrix equation (30) are given below. Primes indicate derivatives of a function. $k_{p}$ and $k_{s}$ indicate wavenumbers of the lowest order compressional and shear guided wave plate modes, while $k_{1}, k_{2}$ and $k_{3}$ are the wavenumbers for flexural waves according to Mindlin theory. $\mathrm{k}_{1 b}, k_{2 b}, k_{3 b}$ are the respective flexural wave wavenumbers in the defect region.

$$
\begin{aligned}
& \alpha_{1,1}=\left(k_{p} r\right) H_{m}^{\prime}\left(k_{p} r\right), \alpha_{1,2}=i m H_{m}\left(k_{s} r\right), \alpha_{1,3}=-\left(1-\frac{b}{h}\right) A_{1 h}\left(k_{1} r\right) H_{m}^{\prime}\left(k_{1} r\right), \\
& \alpha_{1,4}=-\left(1-\frac{b}{h}\right) A_{2 h}\left(k_{2} r\right) H_{m}^{\prime}\left(k_{2} r\right), \alpha_{1,5}=-\left(1-\frac{b}{h}\right) m H_{m}\left(k_{3} r\right), \\
& \alpha_{1,6}=-\left(k_{p} r\right) J_{m}^{\prime}\left(k_{p} r\right), \alpha_{1,7}=-i m J_{m}\left(k_{s} r\right) \\
& \alpha_{2,1}=i m H_{m}\left(k_{p} r\right), \alpha_{2,2}=-\left(k_{s} r\right) H_{m}^{\prime}\left(k_{s} r\right), \alpha_{2,3}=-i m\left(1-\frac{b}{h}\right) A_{1 h} H_{m}\left(k_{1} r\right) \\
& \alpha_{2,4}=-i m\left(1-\frac{b}{h}\right) A_{2 h} H_{m}\left(k_{2} r\right), \alpha_{2,5}=-i\left(1-\frac{b}{h}\right)\left(k_{3} r\right) H_{m}^{\prime}\left(k_{3} r\right), \\
& \alpha_{2,6}=-i m J_{m}\left(k_{p} r\right), \alpha_{2,7}=\left(k_{s} r\right) J_{m}^{\prime}\left(k_{s} r\right) \\
& \alpha_{3,3}=H_{m}\left(k_{1} r\right), \alpha_{3,4}=H_{m}\left(k_{2} r\right), \alpha_{3,8}=-J_{m}\left(k_{1 b} r\right), \alpha_{3,9}=-J_{m}\left(k_{2 b} r\right) \\
& \alpha_{4,3}=A_{1 h}\left(k_{1} r\right) H_{m}^{\prime}\left(k_{1} r\right), \alpha_{4,4}=A_{2 h}\left(k_{2} r\right) H_{m}^{\prime}\left(k_{2} r\right), \alpha_{4,5}=m H_{m}\left(k_{3} r\right), \alpha_{4,8}=-A_{1 b}\left(k_{1 b} r\right) J_{m}^{\prime}\left(k_{1 b} r\right) \text {, } \\
& \alpha_{4,9}=-A_{2 b}\left(k_{2 b} r\right) J_{m}^{\prime}\left(k_{2 b} r\right), \alpha_{4,10}=-m J_{m}\left(k_{3 b} r\right) \\
& \alpha_{5,3}=i m A_{1 h} H_{m}\left(k_{1} r\right), \alpha_{5,4}=i m A_{2 h} H_{m}\left(k_{2} r\right), \alpha_{5,5}=i\left(k_{3} r\right) H_{m}^{\prime}\left(k_{3} r\right) \text {, } \\
& \alpha_{5,8}=-i m A_{1 b} J_{m}\left(k_{1 b} r\right), \alpha_{5,9}=-i m A_{2 b} J_{m}\left(k_{2 b} r\right), \alpha_{5,10}=-i\left(k_{3 b} r\right) J_{m}^{\prime}\left(k_{3 b} r\right) \\
& \alpha_{6,1}=\left(m^{2}-\alpha\left(k_{p} r\right)^{2}\right) H_{m}\left(k_{p} r\right)-\left(k_{p} r\right) H_{m}^{\prime}\left(k_{p} r\right), \alpha_{6,2}=i m\left[\left(k_{s} r\right) H_{m}^{\prime}\left(k_{s} r\right)-H_{m}\left(k_{s} r\right)\right] \text {, } \\
& \alpha_{6,6}=-\left(\frac{b}{h}\right)\left(m^{2}-\alpha\left(k_{p} r\right)^{2}\right) J_{m}\left(k_{p} r\right)-\left(k_{p} r\right) J_{m}^{\prime}\left(k_{p} r\right), \alpha_{6,7}=-i m\left(\frac{b}{h}\right)\left[\left(k_{s} r\right) J_{m}^{\prime}\left(k_{s} r\right)-J_{m}\left(k_{s} r\right)\right] \\
& \alpha_{7,1}=i m\left[\left(k_{p} r\right) H_{m}^{\prime}\left(k_{p} r\right)-H_{m}\left(k_{p} r\right)\right], \alpha_{7,2}=\left[\left(\frac{\left(k_{p} r\right)^{2}}{2}-m^{2}\right) H_{m}\left(k_{s} r\right)+\left(k_{s} r\right) H_{m}^{\prime}\left(k_{s} r\right)\right] \text {, } \\
& \alpha_{7,6}=-i m\left(\frac{b}{h}\right)\left[\left(k_{p} r\right) J_{m}^{\prime}\left(k_{p} r\right)-J_{m}\left(k_{p} r\right)\right], \alpha_{7,7}=-\left(\frac{b}{h}\right)\left[\left(\frac{\left(k_{s} r\right)^{2}}{2}-m^{2}\right) J_{m}\left(k_{s} r\right)+\left(k_{s} r\right) J_{m}^{\prime}\left(k_{s} r\right)\right] \\
& \alpha_{8,2+j}=\frac{2}{3} A_{j h}\left[\left(m^{2}-\alpha\left(k_{j} r\right)^{2}\right) H_{m}\left(k_{j} r\right)-\left(k_{j} r\right) H_{m}^{\prime}\left(k_{j} r\right)\right] j=1,2 \text {, } \\
& \alpha_{8,5}=\frac{2}{3} m\left[\left(k_{3} r\right) H_{m}^{\prime}\left(k_{3} r\right)-H_{m}\left(k_{3} r\right)\right] \text {, }
\end{aligned}
$$




$$
\begin{aligned}
& \alpha_{8,6}=2\left(1-\frac{b}{h}\right)\left(\frac{b}{h}\right)\left[\left(m^{2}-\alpha\left(k_{p} r\right)^{2}\right) J_{m}\left(k_{p} r\right)-\left(k_{p} r\right) J_{m}^{\prime}\left(k_{p} r\right)\right], \\
& \alpha_{8,7}=2 i m\left(1-\frac{b}{h}\right)\left(\frac{b}{h}\right)\left[\left(k_{s} r\right) J_{m}^{\prime}\left(k_{s} r\right)-J_{m}\left(k_{s} r\right)\right], \\
& \alpha_{8,7+j}=-\frac{2}{3}\left(\frac{b}{h}\right)^{3} A_{j b}\left[\left(m^{2}-\alpha\left(k_{j b} r\right)^{2}\right) J_{m}\left(k_{j b} r\right)-\left(k_{j b} r\right) J_{m}^{\prime}\left(k_{j b} r\right)\right] j=1,2, \\
& \alpha_{8,10}=-\frac{2}{3}\left(\frac{b}{h}\right)^{3} m\left[\left(k_{3 b} r\right) J_{m}^{\prime}\left(k_{s b} r\right)-J_{m}\left(k_{3 b} r\right)\right], \\
& \alpha_{9,2+j}=i m \frac{2}{3} A_{j h}\left[\left(k_{j} r\right) H_{m}^{\prime}\left(k_{j} r\right)-H_{m}\left(k_{j} r\right)\right] j=1,2, \\
& \alpha_{9,5}=i \frac{2}{3}\left[\left(m^{2}-\frac{\left(k_{3} r\right)^{2}}{2}\right) H_{m}\left(k_{3} r\right)-\left(k_{3} r\right) H_{m}^{\prime}\left(k_{3} r\right)\right], \alpha_{9,6}=2 i m\left(1-\frac{b}{h}\right)\left(\frac{b}{h}\right)\left[\left(k_{p} r\right) J_{m}^{\prime}\left(k_{p} r\right)-J_{m}\left(k_{p} r\right)\right], \\
& \alpha_{9,7}=2\left(1-\frac{b}{h}\right)\left(\frac{b}{h}\right)\left[\left(\frac{\left(k_{s} r\right)^{2}}{2}-m^{2}\right) J_{m}\left(k_{s} r\right)+\left(k_{s} r\right) J_{m}^{\prime}\left(k_{s} r\right)\right], \\
& \alpha_{9,7+j}=-i m \frac{2}{3}\left(\frac{b}{h}\right)^{3} A_{j b}\left[\left(k_{j b} r\right) J_{m}^{\prime}\left(k_{j b} r\right)-J_{m}\left(k_{j b} r\right)\right], j=1,2, \\
& \alpha_{10,7+j}=-\left(\frac{b}{h}\right)\left(1+A_{j b}\right)\left(k_{j b} r\right) J_{m}^{\prime}\left(k_{j b} r\right), j=1,2, \alpha_{10,10}=-\left(\frac{b}{h}\right) m J_{m}\left(k_{3 b} r\right) . \\
& \alpha_{9,10}=-i \frac{2}{3}\left(\frac{b}{h}\right)^{3}\left[\left(m^{2}-\frac{\left(k_{3 b} r\right)^{2}}{2}\right) J_{m}\left(k_{3 b} r\right)-\left(k_{3 b} r\right) J_{m}^{\prime}\left(k_{3 b} r\right)\right],
\end{aligned}
$$

\section{$\underline{\text { S0 incidence }}$}

$$
\begin{aligned}
& \beta_{1}=-i^{m}\left(k_{p} r\right) J_{m}^{\prime}\left(k_{p} r\right), \beta_{2}=-m i^{m+1} J_{m}\left(k_{p} r\right), \\
& \beta_{7}=-i^{m}\left[\left(m^{2}-\alpha\left(k_{p} r\right)^{2}\right) J_{m}\left(k_{p} r\right)-\left(k_{p} r\right) J_{m}^{\prime}\left(k_{p} r\right)\right], \beta_{8}=-m i^{m+1}\left[\left(k_{p} r\right) J_{m}^{\prime}\left(k_{p} r\right)-J_{m}\left(k_{p} r\right)\right] .
\end{aligned}
$$




\section{REFERENCES}

[1] P. Fromme, P. Wilcox, M.J.S. Lowe and P. Cawley, “On the development and testing of a guided ultrasonic wave array for structural integrity monitoring”, IEEE Trans. Ultra. Fer. Freq. Cont. 53, 777-786 (2006).

[2] C.H. Wang and L.R.F. Rose, "Plate-Wave diffraction tomography for structural health monitoring”, Review of QNDE Vol. 22, edited by D.O Thompson and D.E. Chimenti, 1615-1622, (2003), AIP, New York

[3] K.R. Leonard, E.V. Malyarenko and M.K. Hinders, "Ultrasonic Lamb wave tomography”, Inverse Problems 18, 1795-1808 (2002).

[4] O. Diligent, T. Grahn, A. Boström, P. Cawley and M. J. S. Lowe, “The low-frequency reflection and scattering of the S0 Lamb mode from a circular through-thickness hole in a plate: Finite Element, analytical and experimental studies”, J. Acoust. Soc. Am. 112, 25892601 (2002).

[5] O. Diligent and M.J.S. Lowe, "Reflection of the S0 Lamb mode from a flat bottom circular hole”, J. Acoust. Soc. Am. 118, 2869-2879 (2005).

[6] J. C. P. McKeon and M. K. Hinders, "Lamb wave scattering from a through hole”, J. Sou. Vib. 224, 843-862 (1999).

[7] C.H. Wang and F.Chang, "Scattering of plate waves by a cylindrical inhomogeneity", J. Sound Vib. 282, 429-451 (2005).

[8] C. Vemula and A.N. Norris, "Flexural wave propagation and scattering on thin plates using Mindlin theory”, Wave Motion 26, 1-12 (1997).

[9] P. Fromme and M.B. Sayir, "Measurement of the scattering of a Lamb wave by a through hole in a plate”, J. Acoust. Soc. Am. 111, 1165-1170 (2002).

[10] T. Grahn, "Lamb wave scattering from a circular partly through-thickness hole in a plate”, Wave Motion 37, 63-80 (2003).

[11] G. Konstantinidis, B. W. Drinkwater and P. D. Wilcox, "The Long Term Stability of Guided Wave Structural Health Monitoring Systems”, AIP Conf. Proc. 820,1702-1710 (2006).

[12] J. Hou, K.R. Leonard and M.K. Hinders, “Automatic Multi-mode Lamb Wave Arrival Time Extraction for Improved Tomographic Reconstruction”, Inv. Prob. 20, 1873-1888 (2004)

[13] J. D. Achenbach, Wave propagation in elastic solids, North-Holland, Amsterdam (1973).

[14] K.F. Graff, Wave motion in elastic solids, Clarendon Press, Oxford (1975). 
[15] O. Diligent and M.J.S. Lowe, "Prediction and measurement of nonpropagating Lamb modes at the free end of a plate when the fundamental antisymmetric mode A0 is incident", J. Acoust. Soc. Am. 113, 3032-3042 (2003).

[16] P. Fellinger, R. Marklein, K.J. Langenberg and S. Klaholz, „Numerical modeling of elastic wave propagation and scattering with EFIT- elastodynamic finite integration technique." Wave Motion 21, 47-66 (1995) 